\title{
Sequential transformations of human sperm nucleus in human egg
}

\author{
B. Lassalle and J. Testart \\ INSERM Unité 187, 32 rue des Carnets, 92141 Clamart, France
}

\begin{abstract}
Summary. In-vitro insemination of human zona-free oocytes prepared from oocytes that failed to fertilize in an in-vitro fertilization programme was used as an experimental model to study the time course and morphological events during the development of sperm nuclei into male pronuclei. At $30 \mathrm{~min}$ after insemination, 22 eggs were cultured in a $\mathrm{CO}_{2}$ incubator for further $3.5 \mathrm{~h}$ and 17 eggs were placed individually between a slide and coverslip for randomly repeated microscopical observations in a controlled environment for at least $3.5 \mathrm{~h}$. Simultaneous arrest of maternal meiosis and sperm nuclear development occurred in $36.4 \%(8 / 22)$ eggs cultured in the $\mathrm{CO}_{2}$ incubator and $47.1 \%(8 / 17)$ of those cultured between a slide and coverslip. Sequential transformation of the human sperm nucleus in human eggs was studied in 6 eggs that showed continuous development of sperm nuclei into male pronuclei during at least $3.5 \mathrm{~h}$ after insemination. The early sperm nuclear development in human egg ooplasm can be divided into three phases: the sperm nucleus first decondenses (phase 1) then partly recondenses (phase 2 ) before expanding again to form an early male pronucleus (phase 3). The prepronuclear stages (phases 1 and 2) took about $60 \mathrm{~min}$ each and the pronuclear formation (phase 3) began between 120 and $170 \mathrm{~min}$ after insemination. Early pronuclear formation was associated with the occurrence of dense outline material, probably a precursor of the future pronuclear membrane, around the recondensed nucleus in re-expansion (phase 3). Between 30 and $60 \mathrm{~min}$ after the beginning of phase 3, numerous ( $>20$ ) dense grains, considered as nucleolar precursors, were clearly visible inside the growing male pronucleus. Moreover, we have examined sperm nuclear changes in some eggs in which the progression of late meiosis was abnormal. Meiotic arrest of maternal chromatin was always associated with arrest of sperm head development. In $75 \%(6 / 8)$ of the eggs arrested in the metaphase II stages and in $87.5 \%$ $(7 / 8)$ of the eggs arrested in late anaphase II, sperm nuclear development was stopped at the decondensed and recondensed stages, respectively. We have always observed male pronuclei when a maternal pronucleus was present in the egg. These observations suggested that maternal chromatin and sperm nuclear development are probably regulated by common factor(s).
\end{abstract}

Keywords: man; egg; polyspermy; sperm nuclear development; pronuclear membrane; pronuclei formation

\section{Introduction}

Sperm nuclear decondensation and male pronuclear formation are the first events after spermoocyte fusion that permit male contribution to the embryonic genome. According to the literature, there are two steps in sperm nuclear development, i.e. sperm nuclear decondensation (for review, see Zirkin et al., 1985) and male pronuclear formation (hamster: Austin, 1951; rat: Austin \& Walton, 1960). Tesarik \& Kopecny (1989a) have defined four stages of sperm nuclear development in humans. The first two stages, representing partial (stage 1) and total (stage 2) sperm nuclear 
decondensation, appeared as early as $1 \mathrm{~h}$ after mixing of gametes. Sperm chromatin decondensation concerns sperm nuclear envelope breakdown, decondensation of sperm chromatin mediated by partial hydrolytic degradation, disulphide bond reduction of sperm nuclear proteins, and replacement of sperm protamines by egg histones (for review, see Zirkin et al., 1985). The early stage of male pronucleus formation (stage 3 ) was characterized by reformation of a nuclear envelope, reorganization of chromatin, and the assembly of nucleolar precursors. The fully developed male pronucleus (stage 4) appears no earlier than $12 \mathrm{~h}$ after insemination. This last stage was characterized by the completion of nucleolar precursor development, further changes in chromatin distribution, modification of the nuclear envelope (Tesarik \& Kopecny, 1989a) and the onset of the DNA synthetic phase (Tesarik \& Kopecny, 1989b). In the hamster, sperm nuclear development has been described as consisting of three successive stages, i.e. sperm nuclear decondensation, followed by sperm nuclear condensation and, finally, sperm pronuclear formation (Wright \& Longo, 1988).

In this study, the process of human sperm nuclear development was analysed with the use of an experimental model based on in-vitro insemination of human zona-free oozytes prepared from oocytes that failed to fertilize in a clinical in-vitro fertilization programme. We describe the main morphological changes and the time course of human sperm nucleus transformation in mature, although in-vitro aged, human oocytes. The observations were made up to the early pronucleus stage by frequently repeated microscopic examinations in a controlled environment. Moreover, we have examined sperm nuclear changes in some eggs in which the progression of late meiosis was abnormal.

\section{Materials and Methods}

Preparation of zona-free human oocytes. Human oocytes that had failed to be fertilized by $40-48 \mathrm{~h}$ after in-vitro insemination in our in-vitro fertilization programme were used in this study. Details of the procedures have been published elsewhere (Testart et al., 1989). The experiments were approved by the National Ethical Committee. Oocytes were observed under an inverted microscope before zona digestion. All the 39 oocytes analysed were mature and showed no signs of fertilization. Enzymic digestion of the zona pellucida was performed following the method described by Tesarik \& Kopecny (1989a) and slightly modified as follows. Unfertilized oocytes were incubated in $0 \cdot 1 \%(w / v)$ pronase (Sigma, St Louis, MO, USA), dissolved in Dulbecco's phosphate-buffered saline (PBS: Gibco, Cergy-Pontoise, France) and zona digestion was controlled microscopically. When the zona pellucida became soft and undulated (after about $5 \mathrm{~min}$ ), the oocytes were rapidly transferred into B2 medium (B2 Menezo: Api System, La Balme les Grottes, France) plus $0.4 \%$ (w/v) bovine serum albumin (BSA: Fraction V, Sigma) and incubated for $1-10 \min \left(37^{\circ} \mathrm{C}, 5 \% \mathrm{CO}_{2}\right)$ before transfer into insemination medium.

Preparation of insemination medium. Frozen spermatozoa from one donor's ejaculate allowed the use of 22 straws for successive assays. The cryoprotectant medium used and the freezing method were as described by Jondet (1976). Sperm thawing was done at room temperature and straw content $(0.25 \mathrm{ml})$ was diluted with $5 \mathrm{ml}$ PBS plus $0.4 \%(\mathrm{w} / \mathrm{v})$ BSA (PBS-BSA medium). The sperm suspension was centrifuged at $600 \mathrm{~g}$ for $7 \mathrm{~min}$ and the pellet was resuspended in $1 \mathrm{ml}$ B2-BSA medium to constitute the insemination medium with a final motile sperm concentration of $0.4-0.5 \times 10^{6}$ spermatozoa/ml. Zona-free human oocytes $(1-4)$ were placed in insemination medium into dishes and the culture was performed in a $\mathrm{CO}_{2}$ incubator $\left(37^{\circ} \mathrm{C}, 5 \% \mathrm{CO}_{2}\right)$.

Egg culture and measurement of sperm nuclear development. At $30 \mathrm{~min}$ after insemination, the oocytes were rinsed in fresh B2-BSA medium and 22 eggs were replaced in the $\mathrm{CO}_{2}$ incubator for incubation for a further $4 \mathrm{~h}$ in $\mathrm{B} 2$-BSA medium, and 17 eggs were placed individually between a slide and a coverslip in B2-BSA medium for repeated microscopical observations during at least $4 \mathrm{~h}$. Small amounts of Vaseline were applied on the slide at four points around the droplet containing the specimen before the coverslip was placed over and pressed slightly downwards until oocyte structures could be clearly seen. Slide and coverslip were sealed together with paraffin wax. These manipulations took less than 2 min and the preparation was placed under a phase-contrast microscope $(\times 1000)$ inside a modified incubator for newborn babies (Lassalle \& Testart, 1988). The temperature was regulated at $37 \cdot 0 \pm 0 \cdot 2^{\circ} \mathrm{C}$ during the time of oocyte culture between slide and coverslip.

From the beginning of sperm nuclear decondensation, the smallest and the largest diameters of the sperm nucleus were measured with the use of an ocular micrometer at the equatorial focal plane. Measurements were also performed on 10 intact sperm heads bound to the oocyte surface. The surface of equatorial sections of decondensing or intact sperm heads was calculated as an elliptical area (elliptical area $=\pi \mathrm{ab}$, with $\mathrm{a}=$ small radius and $\mathrm{b}=$ large radius). For each egg, 10-17 measurements were performed during $0.5-5.5 \mathrm{~h}$ after insemination.

All the eggs were polyspermic ( $2-10$ decondensing sperm heads per oocyte) and several sperm heads could be identified and measured in the same egg. However, to be sure that sequential measurements were done on the same 
sperm heads, only $1-3$ sperm heads were taken into account. These analysed sperm heads were those which decondensed first in the ooplasm. Hence the regulation of sperm decondensation was studied according to the rank in time of the first sperm heads (1-3) that decondensed in each egg. The following values were measured: (A) rate of progression in size $\left(\mu \mathrm{m}^{2} / \mathrm{min}\right)$, i.e. mean of slopes of regression equations; (B) maximal area $\left(\mu \mathrm{m}^{2}\right)$ reached by sperm heads; (C) time after insemination to reach maximal size ( $\mathrm{min}$ ); and (D) estimated time ( $\mathrm{min}$ ) for the sperm head decondensation process, i.e. (maximal area (B) - intact sperm area)/A, with intact sperm head area $=$ $11.2 \pm 0.24 \mathrm{~m}^{2}$. The regulation of sperm pronuclear formation was also studied according to the rank in time of the first $(1-3)$ decondensing sperm heads. Three parameters were studied: (E) the rate of pronucleus progression in size $\left(\mu \mathrm{m}^{2} / \mathrm{min}\right)$; (F) pronuclear area $\left(\mu \mathrm{m}^{2}\right)$ when nucleoli were first observed inside the pronucleus; and $(\mathrm{G})$ the time after insemination ( $\mathrm{min})$ for starting of $F$.

Statistical methods. Data were analysed using Stat View II software provided by a Macintoch II $\times$ computer. Regression equations were obtained and linearity was tested using F tests. Means were expressed with the standard error of the means and compared using Student's $t$ test.

\section{Results}

\section{Prepronuclear development of human sperm head}

Using living gametes sperm-oocyte fusion cannot be detected microscopically and the start of sperm nuclear decondensation is the first evidence for sperm penetration into the egg vitellus. The start of sperm head decondensation became apparent when the outline of the sperm head became vague and when the opacity of the sperm nucleus decreased progressively. These two modifications appeared first around the anterior region of the sperm head, then they gradually reached the posterior part of the head. The sperm head swelled progressively (Fig. 1) and assumed an oval and somewhat rounded form. In some cases, a small dense mass (probably a remaining chromatin mass) persisted for several minutes at the posterior region of the spem head (Fig. 1a). The size of sperm nucleus was maximal at the end of the decondensing process (Fig. 1b,c) then it decreased progressively until pronucleus formation (Fig. 1d).

There were 5-20 spermatozoa bound to the vitellus surface by $30 \mathrm{~min}$ after mixing of gametes. In $68.8 \%$ ( 11 out of 16 eggs), at least one spermatozoon had already begun the nuclear decondensation process by $60 \mathrm{~min}$ after insemination. Polyspermic penetration was spread over several hours and finally all the eggs became polyspermic by $3 \mathrm{~h}$ after insemination.

Since sperm head decondensation started at different times in individual observed eggs, it was put in relation not only to the time after insemination but also to that of maximal decondensing sperm bead area $\left(t_{\max }=0 \mathrm{~min}\right)$. This allowed limitation of the effects of different delays in sperm-egg fusion amongst the eggs in the time course of sperm head development process. During the sperm decondensation phase (Fig. 2), the area of the sperm head expanded linearly in time according to time after insemination (correlation coefficient $(r)=0.42, P<0.03)$ or $t_{\max }(r=0.58$, $P<0.0001)$. It increased $(P<0.001)$ about 7 -fold in about $60 \mathrm{~min}$ in our culture conditions (Table 1). After sperm head decondensation, the area of the swollen sperm head regressed linearly in time according to time after insemination $(r=0.38, P<0.002)$ or $\left.t_{\max }{ }^{\prime} r=0.65, P<0.0001\right)$ (Fig. 2) and reached a minimal surface at $41-60 \min (P<0.001)$ (Table 1). The minimal area of the recondensed sperm heads was always greater than that of intact spermatozoa $(P<0.001)$. At this stage of development, the recondensed sperm head always had a rounded form. The recondensation phase was estimated to take about $60 \mathrm{~min}$ in our culture conditions (Table 1).

There were no significant differences in sperm head decondensation characteristics in relation to the rank in time of decondensing sperm heads (Table 2). However, when compared to the first head, the rate of progression in size was 1.4-fold and 1.8-fold increased for the second and the third decondensing sperm head, respectively. The sperm head decondensation phase was more rapidly completed for the third and second than for the first decondensing sperm head (Table 2). Variations between eggs were observed the measures for A (range 0.95-7.65 $\left.\mu \mathrm{m}^{2} / \mathrm{min}\right), B\left(\right.$ range 67.6-95.6 $\mu \mathrm{m}^{2}$ ), $\mathrm{C}(57 \cdot 0-125 \cdot 0 \mathrm{~min})$ and $\mathrm{D}(10 \cdot 7-74 \cdot 5 \mathrm{~min})$. 

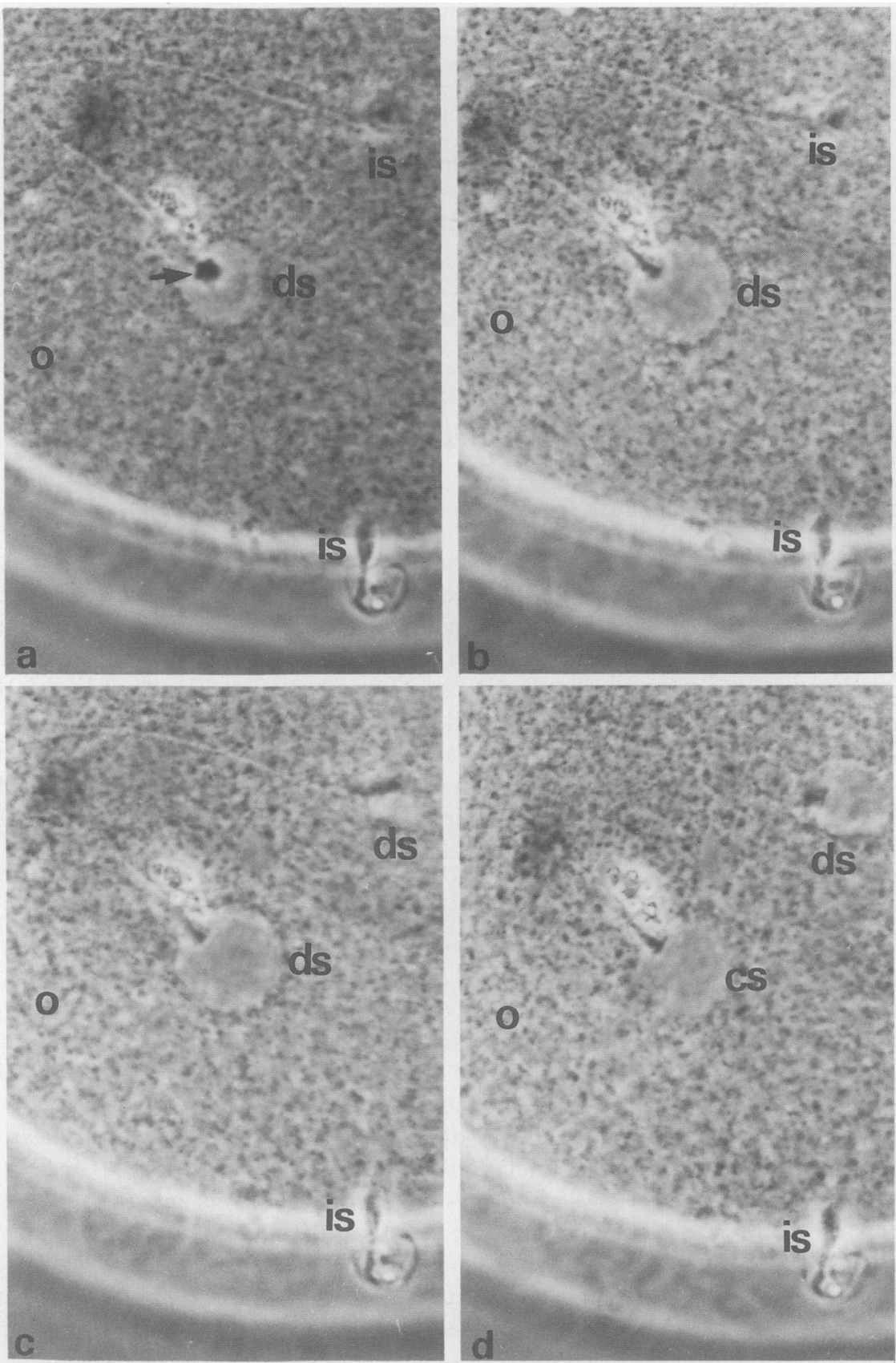

Fig. 1. Prepronuclear development of the human sperm head. First decondensing sperm head 59 min after insemination (a). Small dense mass (probably remaining chromatin mass) persisted for several minutes (arrow). First decondensing sperm head progessively swell (b) to reach maximal size $69 \mathrm{~min}$ after insemination (c). At this time, the second sperm head began to decondense (c). Then, first and second decondensing sperm heads regressed and increased in size 79 min after insemination, respectively (d). Phase contrast. $\times 1000$. 


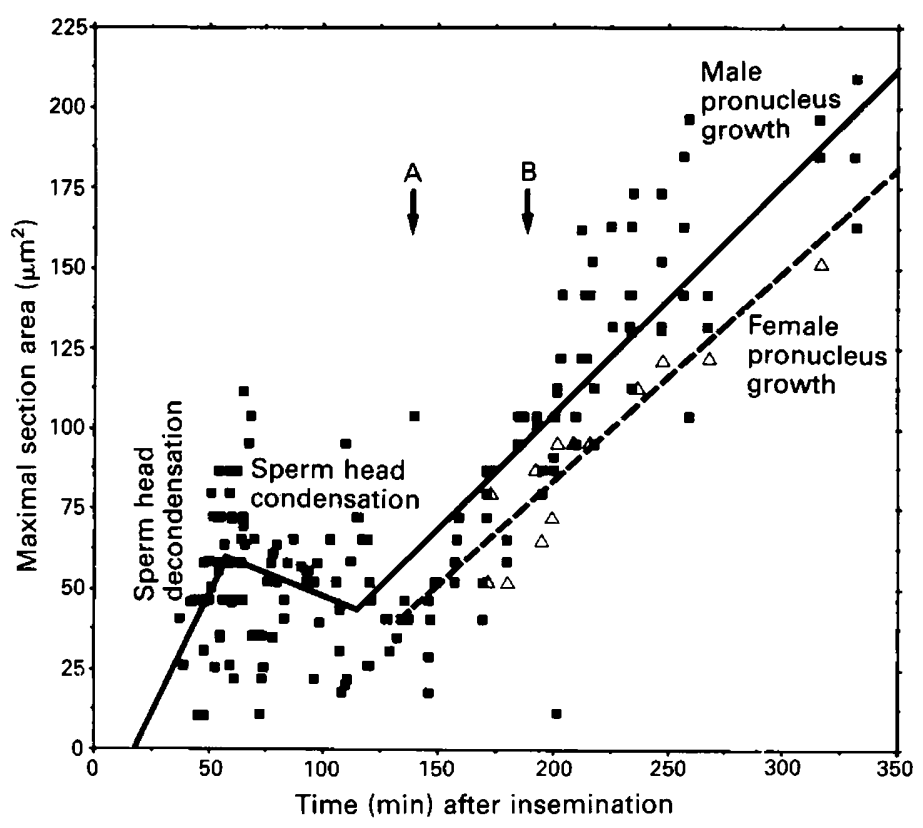

Fig. 2. Male nuclear-pronuclear $(\square)$ and female pronuclear $(\triangle)$ development (maximal section area) according to the time after insemination (6 eggs as indicated in Table 1). (a) First time of appearance of outline around sperm heads. (b) First time of appearance of nucleoli precursors in male pronuclei.

Table 1. Area of human sperm heads related to the time of maximal enlargement (time 0 ) in 6 human oocytes cultured between a slide and coverslip

\begin{tabular}{cccccc}
\hline Time (min) & $\begin{array}{c}\text { Sperm head } \\
\text { area } \pm \text { s.e.m. } \\
\left(\mu \mathrm{m}^{2}\right)^{*}\end{array}$ & $\begin{array}{c}\text { No. of } \\
\text { decondensed } \\
\text { heads }\end{array}$ & Time (min) & $\begin{array}{c}\text { Sperm head } \\
\text { area } \pm \text { s.e.m. } \\
\left(\mu \mathrm{m}^{2}\right)^{*}\end{array}$ & $\begin{array}{c}\text { No. of } \\
\text { decondensed } \\
\text { heads }\end{array}$ \\
\hline & $11 \cdot 2 \pm 0 \cdot 24^{\text {a,c }}$ & 10 & 61 to 80 & $51 \cdot 4 \pm 9 \cdot 6$ & 6 \\
-60 to -40 & $18 \cdot 1 \pm 7 \cdot 3$ & 2 & 81 to 100 & $65 \cdot 5 \pm 13 \cdot 1$ & 8 \\
-39 to -20 & $48 \cdot 0 \pm 2 \cdot 1$ & 3 & 101 to 120 & $89 \cdot 5 \pm 7 \cdot 0$ & 5 \\
-19 to 0 & $58 \cdot 3 \pm 3 \cdot 8$ & 37 & 121 to 140 & $103 \cdot 2 \pm 1 \cdot 0$ & 3 \\
0 & $76 \cdot 7 \pm 5 \cdot 0^{\text {a,b }}$ & 10 & 141 to 160 & $124 \cdot 8 \pm 5 \cdot 2$ & 7 \\
1 to 20 & $53 \cdot 1 \pm 4 \cdot 0$ & 21 & 161 to 180 & $135.9 \pm 8 \cdot 2$ & 5 \\
21 to 40 & $46 \cdot 7 \pm 5 \cdot 1$ & 7 & 181 to 200 & $124 \cdot 6 \pm 9 \cdot 2$ & 5 \\
41 to 60 & $32 \cdot 6 \pm 4 \cdot 0^{\text {b.c.d }}$ & 9 & 201 to 220 & $162 \cdot 1 \pm 29 \cdot 1^{\text {d }}$ & 3 \\
\hline
\end{tabular}

a,b $P<0.0001 ;{ }^{c} P<0.001 ;{ }^{d} P<0.01$.

*Fifteen spermatozoa were examined (2-3 per egg); there were 10-17 measurements for each studied spermatozoon randomly distributed over $0.5-5.5 \mathrm{~h}$ after insemination.

\section{Male pronuclear formation}

The onset of re-expansion of the secondarily condensed sperm head that occurred in eggs at the late anaphase II stage (Fig. 3a) was accompanied by the appearance of a dense outline (Fig. 3b) around the condensed sperm nucleus. This was considered as the beginning of pronuclear formation (Fig. 2). This dense outline that appeared about $120 \mathrm{~min}$ after the beginning of sperm head decondensation (Fig. 2) was conserved around the developing pronucleus and already constituted the outline of the future pronucleus. About $30-60 \mathrm{~min}$ after the outline formation, numerous $(>20)$ 
Table 2. Regulation of sperm head decondensation according to the rank in time of decondensing sperm heads in 15 oocytes

\begin{tabular}{|c|c|c|c|}
\hline \multirow{2}{*}{$\begin{array}{l}\text { Measures of sperm head } \\
\text { decondensation }\end{array}$} & \multicolumn{3}{|c|}{ Rank of decondensing sperm heads into the eggs } \\
\hline & First & Second & Third \\
\hline $\begin{array}{l}\text { A: rate of progression in size } \\
\left(\mu \mathrm{m}^{2} / \mathrm{min}\right) \ddagger\end{array}$ & $2.44 \pm 0.48(15)$ & $3.40 \pm 0.81(9)$ & $4 \cdot 28 \pm 1 \cdot 17(5)$ \\
\hline $\begin{array}{l}\text { B: maximal area reached by } \\
\text { decondensing sperm head }\left(\mu \mathrm{m}^{2}\right)\end{array}$ & $82 \cdot 1 \pm 3 \cdot 0(15)$ & $80 \cdot 4 \pm 4 \cdot 5(9)$ & $82 \cdot 5 \pm 6 \cdot 2(5)$ \\
\hline $\begin{array}{l}\text { C: time after insemination to reach } \\
\text { maximal sperm head area (min) }\end{array}$ & $77 \cdot 1 \pm 6 \cdot 4(15)$ & $79 \cdot 6 \pm 8 \cdot 0(9)$ & $75 \cdot 2 \pm 8 \cdot 0(5)$ \\
\hline $\begin{array}{l}\text { D: estimated time for sperm head } \\
\text { decondensation (min) }\end{array}$ & $29 \cdot 1 \pm 5 \cdot 8(15)$ & $20 \cdot 4 \pm 5 \cdot 3(9)$ & $16 \cdot 7 \pm 5 \cdot 1(5)$ \\
\hline
\end{tabular}

Values are mean \pm s.e.m. for the numbers of spermatozoa observed indicated in parentheses.

$†$ Mean of slopes of regression equations. In one case the second and the third sperm heads reached the maximum sizes at the same time.

dense and small grains (Fig. 3c) were observed inside the growing pronucleus (Fig. 2). The male pronucleus expanded linearly in time after insemination $(r=0.83, P<0.0001)$ and $t_{\max }(r=0.84$, $P<0.0001$ ) (Fig. 2), a $5 \cdot 0$-fold increase by $220 \mathrm{~min}$ (Table 1). There were no significant differences in measures of sperm pronuclear formation in relation to the rank of each of the three first decondensing sperm heads (Table 3).

\section{Relation between sperm head development and meiotic maturation of maternal chromatin}

Simultaneous arrest of maternal meiosis and sperm nuclear development occurred in 8 out of 22 oocytes $(36.4 \%)$ incubated in the $\mathrm{CO}_{2}$ incubator and in 8 out of 17 oocytes $(47.1 \%)$ of those cultured between a slide and coverslip (no significant difference). It was not observed whether the meiotic apparatus of the eggs remained at the metaphase II stage or progressed to the anaphase II stage during the sperm head decondensation phase. However, from 8 eggs remaining at the metaphase II stage after $4 \mathrm{~h}$ in culture, sperm head development was stopped at the decondensation stage in 6 eggs $(75 \%)$ or at recondensation stages in 2 eggs $(25 \%)$. In 7 of 8 eggs $(87.5 \%)$ arrested in anaphase II the sperm heads were stopped at the recondensation stage. In such cases the sperm head areas corresponded to the maximal or minimal means of sperm head areas observed in other eggs. Area of the male pronucleus increased concomitantly (time after insemination, $r=0 \cdot 83$, $\left.P<0.0001 ; t_{\max }, r=0.96, P<0.0001\right)$ in relation to development of the maternal pronucleus (Fig. 2). Male pronuclei were observed in all the 20 eggs showing a female pronucleus after $4 \mathrm{~h}$ in culture. Of 3 eggs with several small size female pronuclei (fragmented pronucleus), 2 contained male pronuclei and one contained several recondensed sperm heads in the ooplasm.

\section{Discussion}

Human oocytes from in-vitro fertilization attempts have already been used in several experimental studies (Soupart \& Strong, 1975; Nishimoto et al., 1982; Malter \& Cohen, 1989; Sathananthan et al., 1989; Tesarik \& Kopecny, 1989a, b). In our study, arrest of maternal meiotic maturation at metaphase II or anaphase II stages concerned $41.0 \%$ (16 out of 39 ) of the reinseminated eggs cultured either in $\mathrm{CO}_{2}$ incubator or between a slide and coverslip. The use of human oocytes aged in insemination culture medium may have some consequences on zygote development. Arrest of development could also be a consequence of the very high number of sperm heads which are present in the egg. Tesarik \& Kopecny (1989c) have reported an impairment of male pronuclear 

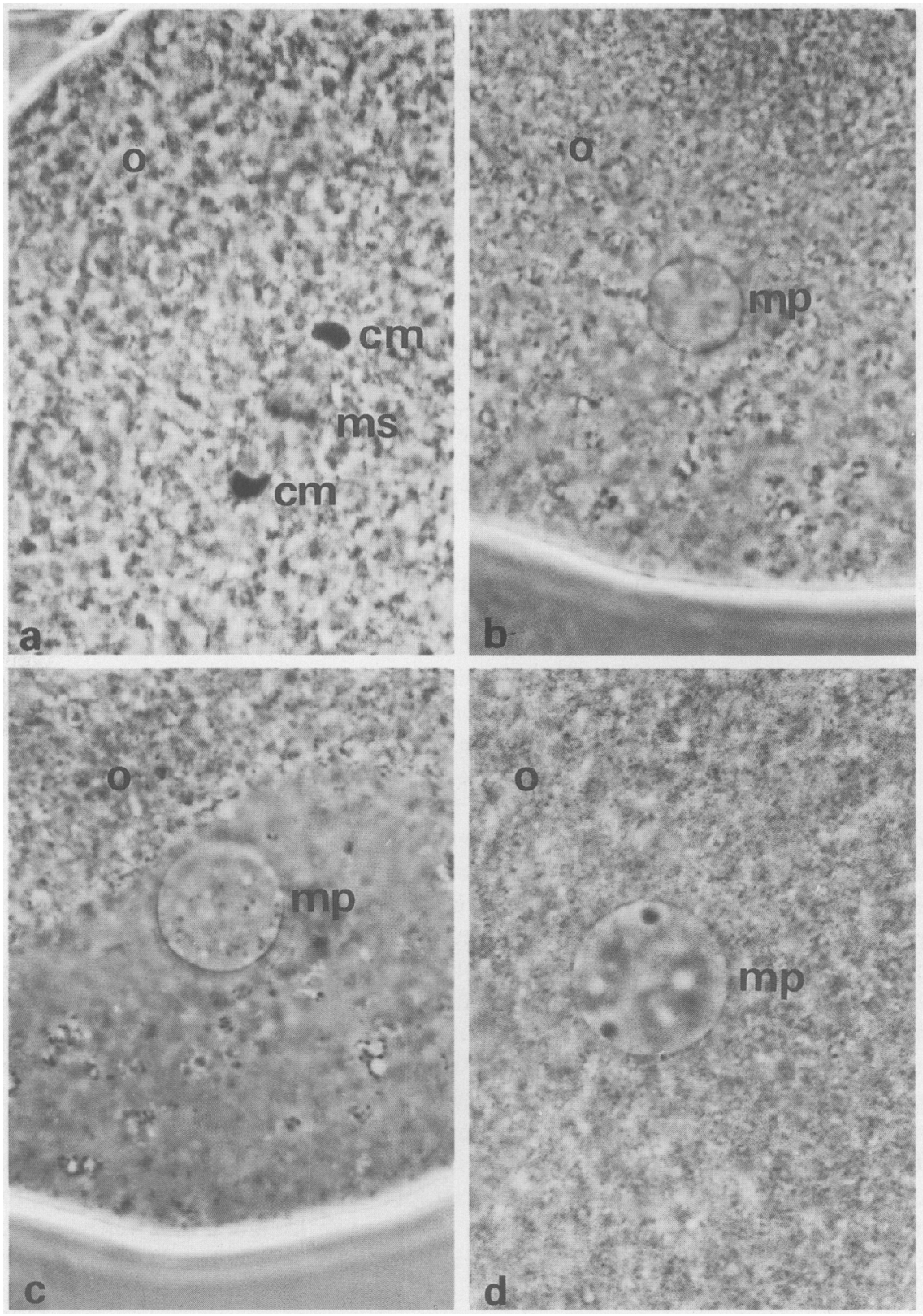

Fig. 3. Male pronuclear formation. (a) In the human egg at late anaphase II stage, a condensed sperm head began to re-expand and a dense outline appeared (120 min after insemination) around the condensed sperm nucleus (b). About 30-60 min after the outline formation, numerous $(>20)$ dense grains were observed inside the growing male pronucleus (c). A male pronucleus was observed after $4 \mathrm{~h}$ of culture (d). Phase contrast, $\times 1000$.

formation with increasing nucleocytoplasmic ratio in humans. In polyspermic mouse eggs either all or only some sperm heads had completed the process of transformation, while the remainder were arrested at the early stage of pronuclear development (Witkowska, 1981). In our study, effects of multiple sperm penetration on sperm nuclear development were only analysed for the first 3 
Table 3. Regulation of male pronuclear formation according to the rank in time of decondensing sperm heads in 7 oocytes

\begin{tabular}{lccc}
\hline \multirow{2}{*}{$\begin{array}{l}\text { Measures of sperm } \\
\text { pronuclear formation }\end{array}$} & \multicolumn{3}{c}{ Rank of decondensing sperm heads into the eggs } \\
\cline { 2 - 4 } & First & Second & Third \\
\hline $\begin{array}{l}\text { E: rate of progression in size } \\
\left(\mu \mathrm{m}^{2} / \mathrm{min}\right) \dagger\end{array}$ & $0.91 \pm 0.07(7)$ & $0.78 \pm 0.07(5)$ & $0.82 \pm 0.05(3)$ \\
$\begin{array}{l}\text { F: pronuclear area when nucleoli } \\
\text { were first time observed }\left(\mu \mathrm{m}^{2}\right)_{+}^{\dagger}\end{array}$ & $97.9 \pm 4.0(4)$ & $98.7 \pm 5.5 \quad(3)$ & $102.8 \pm 1.45(2)$ \\
$\begin{array}{l}\text { G: time after insemination of first } \\
\text { appearance of nucleoli inside male } \\
\text { pronuclei (min) }\end{array}$ & $178.0 \pm 13.2(4)$ & $192.7 \pm 4.3 \quad(3)$ & $189.5 \pm 3.5$ (2) \\
\hline
\end{tabular}

Values are mean \pm s.e.m. for the numbers of pronuclei observed indicated in parentheses.

$\dagger$ Mean of slopes of regression equations.

fObservations in only 4 eggs.

spermatozoa that decondensed in the ooplasm and all spermatozoa finally attained comparable developmental stages in the egg. Egg culture may be considered as suboptimal when performed between a slide and coverslip. However, arrest of both maternal meiosis and sperm head development affected $47 \cdot 1 \%$ of eggs cultured between a slide and coverslip compared with $36 \cdot 4 \%$ of those cultured in $\mathrm{CO}_{2}$ incubator (not significant). Moreover, in eggs cultured in a $\mathrm{CO}_{2}$ incubator or between a slide and coverslip, the areas of male pronuclei were not different $\left(148.6 \mu \mathrm{m}^{2}\right.$ versus $136.0 \mathrm{~m}^{2}$, respectively) $4 \mathrm{~h}$ after sperm insemination (data not shown). Consequently, developmental arrest after this short time period (several hours) cannot be due only to suboptimal culture conditions.

Austin (1951) and Austin \& Walton (1960) have reported the use of in-vitro culture between a slide and coverslip for continuous observations under a phase-contrast microscope to study the cytoplasmic and nuclear changes which occur during fertilization of mammalian eggs. Using comparable conditions with mature but aged human oocytes, we found that sperm head development can be divided into three phases: the sperm head first enlarges (1) then partly recondenses (2) before expanding again to form an early male pronucleus (3). The expansion of human sperm chromatin involves the entire decondensing sperm head as shown by several ultrastructural studies (Lung, 1972; Evenson et al., 1978; Sathananthan et al., 1986). Consequently, the section area of the decondensing sperm head could be considered in relation to the total chromatin mass in expansion. The present study shows that sperm nuclear decondensation phase is completed in about $1 \mathrm{~h}$ in humans. Sperm nuclear recondensation which occurs between sperm nuclear decondensation and pronuclear formation has been described for the surf clam (Luttmer \& Longo, 1988), mouse (Ecklund \& Levine, 1975) and hamster (Naish et al., 1987; Wright \& Longo, 1988). In humans, sperm chromatin recondensed in about $1 \mathrm{~h}$ after the end of the nuclear decondensation phase and the resulting chromatin mass took always a rounded form. In our study, the time course and maximal sperm . head size for completion of the sperm head decondensation phase varied between eggs. Several studies have shown that mammalian eggs contain specific factors implicated in the sperm decondensation process (Usui \& Yanagimachi, 1976; Gall \& Ohsumi, 1976; Wiesel \& Schultz, 1981; Perreault et al., 1984; Calvin et al., 1986). These observations emphasized the role of specific egg cytoplasmic factors in the sperm nuclear development process and variations of these factors between eggs. Such ooplasmic factors may act in a different way according to sperm chromatin stability (Huret, 1986; Perreault et al., 1987) which depends on species-specific molecular structure (type of protamines) (Perreault et al., 1988).

Soon after the recondensation phase, sperm and egg chromatin expanded again and a dense outline appeared progressively around the female chromatin mass. In the cell cycle, reconstruction 
of the new nuclear envelope at the surface of the chromatin mass is initiated by the process of lamina reassembly that would appear in the late anaphase and telophase (Gerace et al., 1978). Development of the male pronuclear envelope in human (Soupart \& Strong, 1975) and invertebrate (reviewed by Longo, 1973) eggs begins by aggregation of vesicles along the periphery of the dispersing sperm chromatin. These vesicles may derive from the endoplasmic reticulum (Soupart \& Strong, 1975). In humans, a primitive nuclear envelope surrounding the newly formed male pronucleus was observed $4 \mathrm{~h}$ after insemination and persisted during at least $8 \mathrm{~h}$ until this development was entirely completed (Tesarik \& Kopecny, 1989a). However, there were no observations made between 2 and $4 \mathrm{~h}$ after insemination. In our study, the dense outline appeared between 2 and $2.8 \mathrm{~h}$ after insemination. We assume that the dense outlines observed around both male and female chromatin masses were precursors of the future pronuclear membranes. Between 30 and $60 \mathrm{~min}$ after the dense outline appearance, numerous $(>20)$ dense and small grains (probably precursors of nucleoli) can be observed inside the growing female and male pronuclei. The nucleolar precursor development seems to be incomplete until $12 \mathrm{~h}$ after in-vitro insemination when fully developed male pronuclei were first observed in human eggs (Tesarik \& Kopecny, 1989a). After the prepronuclear outline formation, male and female pronuclei progressed concomitantly as if they were regulated by the same process.

During sperm head decondensation, the stage of maternal chromatin was difficult to assess in our conditions. In the hamster, sperm chromatin decondensation and recondensation was reported to occur in eggs during the anaphase stage of second meiotic division (Wright \& Longo, 1988). In late anaphase II, defined by the presence of two maternal compacted chromatin masses at the extremities of the spindle, we have observed that human sperm chromatin was frequently recondensed and female and male chromatin materials seem to be already in synchrony. In our study, meiotic arrest of maternal chromatin was always associated with arrest of sperm head development. In $75.0 \%$ of the eggs arrested in the metaphase II stage and in $87.5 \%$ of the eggs arrested in late anaphase II stage, sperm nuclear development was stopped at the decondensed and recondensed stages, respectively. Arrest of the female pronucleus was also associated with arrest of male pronuclear development (unpublished observations). Moreover, we have always observed male pronuclei when a maternal pronucleus was present in the egg. As reported for the hamster (Wright \& Longo, 1988) there was a close relation between development of the female and male chromatin in the human egg. These observations suggest that maternal chromatin and sperm nuclear development are probably regulated by common factor(s).

The total process of sperm nuclear decondensation and recondensation takes less than 60 min in the hamster (Wright \& Longo, 1988) compared to $120 \mathrm{~min}$ in humans (present study). Although the human sperm nucleus decondenses in only 10-15 min in hamster eggs (Perreault et al., 1988), this phase took $60 \mathrm{~min}$ in human eggs according to our observations. However, the hamster sperm head decondensed more slowly (45 min: Perreault et al., 1988; $30 \mathrm{~min}$ : Wright \& Longo, 1988) than did the human sperm head (10-15 min: Perrault et al., 1988) in hamster ooplasm. From these observations it appears that the duration of sperm decondensation is strongly modulated by the animal species from which the oocytes were originated. Since sperm nuclear decondensation was more rapidly completed for the third $(17 \mathrm{~min})$ and the second $(20 \mathrm{~min})$ decondensing sperm head than the first one $(29 \mathrm{~min})$, the time after insemination to reach the maximal nuclear size was the same for the 3 observed spermatozoa. An egg regulator mechanism controlling the sperm nuclear decondensation process is probably stimulated after the first sperm-egg fusion and this regulator mechanism could permit synchronization in time of the development of at least the first 3 decondensing sperm nuclei. These results suggest that the oocyte is strongly implicated in regulation of post-fusion events.

We thank Dr Michel Jondet from the cytogenetic laboratory of the Fondation de Recherche en Hormonologie (F.R.H.) for providing the human frozen semen used in this study. 


\section{References}

Austin, C.R. (1951) The formation, growth, and conjugation of the pronuclei in the rat egg. J. roy. Microsc. Soc. 71, 295-306.

Austin, C.R. \& Walton, A. (1960) Fertilization. In Marshall's Physiology of Reproduction, 3rd edn, Vol. 1, part 2, Ch. 10, pp. 310-396. Ed. A. S. Parkes. Longmans, London.

Calvin, H.I., Grosshans, K. \& Blake, E.J. (1986) Estimation and manipulation of glutathione levels in prepuberal mouse ovaries and ova: relevance to sperm nucleus transformation in the fertilized egg. Gamete Res. 14, 265-275.

Ecklund, P.S. \& Levine, L. (1975) Mouse sperm basic nuclear protein, electrophoretic characterization and fate after fertilization. $J$. Cell Biol. 66, 251-262.

Evenson, D.P., Witkin, S.S., de Harven, E. \& Bendich, A. (1978) Ultrastructure of partially decondensed human spermatozoal chromatin. J. Ultrastruct. Res. 63, 178-187.

Gall, W.E. \& Ohsumi, Y. (1976) Decondensation of sperm nuclei in vitro. Expl Cell Res. 102, 349-358.

Gerace, L., Blum, A. \& Blobel, G. (1978) Immunochemical localization of the major polypeptides of the nuclear pore complex-lamina fraction. Interphase and mitotic distribution. J. Cell Biol. 79, 546-566.

Huret, J.L. (1986) Nuclear chromation decondensation of human sperm: a review. Archs Androl. 16, 97-109.

Jondet, M. (1976) Congélation du sperme humain. Bull. Acad. vét. France 49, 373-384.

Lassalle, B. \& Testart, J. (1988) Human sperm injection into the perivitelline space (SI-PVS) of hamster oocytes: effect of pretreatment by calcium-ionophore A23187 and freezing-thawing on the penetration rate and polyspermy. Gamete Res. 20, 301-311.

Longo, F.J. (1973) Fertilization: a comparative ultrastructural review. Biol. Reprod. 9, 149-215.

Lung, B. (1972) Ultrastructure and chromatin disaggregation of human sperm head with thioglycolate treatment. J. Cell Biol. 52, 179-186.

Luttmer, S.J. \& Longo, F.J. (1988) Sperm nuclear transformations consist of enlargement and condensation coordinate with stages of maturation in fertilized Spisula solidissima oocytes. Devl. Biol. 128, 86-96.

Malter, H.E. \& Cohen, J. (1989) Partial zona dissection of the huma oocyte: a nontraumatic method using micromanipulation to assist zona pellucida penetration. Fert. Steril. 51, 139-148.

Naish, S.J., Perreault, S.D., Foehner, A.L. \& Zirkin, B.R. (1987) DNA synthesis in the fertilizing hamster sperm nucleus: sperm template availability and egg cytoplasmic control. Biol. Reprod. 36, 245-253.

Nishimoto, T., Yamada, I., Niwa, K., Nishimura, T. \& Iritani, A. (1982) Sperm penetration in vitro of human oocytes matured in a chemically defined medium. J. Reprod. Fert. 64, 115-119.

Perreault, S.D., Wolff, R.A. \& Zirkin, B.R. (1984) The role of disulfide bond reduction during mammalian sperm nuclear decondensation in vivo. Devl Biol. 101, 160-167.

Perreault, S.D., Naish, S.J. \& Zirkin, B.R. (1987) The timing of hamster sperm nuclear decondensation and male pronucleus formation is related to sperm nuclear disulfide bond content. Biol. Reprod. 36, 239-244.

Perreault, S.D., Barbee, R.R., Elstein, K.H., Zucker, R.M. \& Keefer, C.L. (1988) Interspecies differences in the stability of mammalian sperm nuclei assessed in vivo by sperm microinjection and in vitro by flow cytometry. Biol. Reprod. 39, 157-167.

Sathananthan, A.H., Ng, S.C., Edirinsinghe, R., Ratnam, S.S. \& Wong, P.C. (1986) Human sperm-egg interaction in vitro. Gamete Res. 15, 317-326.

Sathananthan, A.H., Ng, S.C., Trounson, A., Bongso, A., Laws-King, A. \& Ratnam, S.S. (1989) Human microinsemination by injection of single or multiple sperm: ultrastructure. Human Reprod. 4, 574-583.

Soupart, P. \& Strong, P.A. (1975) Ultrastructural observations on polyspermic penetration of zona pellucidafree human oocytes inseminated in vitro. Fert. Steril. 6, 523-537.

Tesarik, J. \& Kopecny, V. (1989a) Development of male pronucleus: ultrastructure and timing. Gamete Res. 24, 135-150.

Tesarik, J. \& Kopecny, V. (1989b) Nucleic acid synthesis and development of human male pronucleus. $J$. Reprod. Fert. 86, 549-558.

Tesarik, J. \& Kopecny, V. (1989c) Developmental control of the human male pronucleus by ooplasmic factors. Human Reprod. 4, 962-968.

Testart, J., Belaisch-Allart, J., Forman, R., Gazengel, A., Strubb, N. \& Frydman, R. (1989) Influence of different stimulation treatments on oocyte characteristics and in vitro fertilizing ability. Human Reprod. 4, 192-197.

Usui, N. \& Yanagimachi, R. (1976) Behavior of hamster sperm nuclei incorporated into eggs at various stages of maturation, fertilization, and early development. J. Ultrastruct. Res. 57, 276-288.

Wiesel, S. \& Schultz, G.A. (1981) Factors which may affect removal of protamine from sperm DNA during fertilization in the rabbit. Gamete Res. 4, 25-34.

Witkowska, A. (1981) Pronuclear development and the first cleavage division in polyspermic mouse eggs. $J$. Reprod. Fert. 62, 493-498.

Wright, S.J. \& Longo, F.J. (1988) Sperm nuclear enlargement in fertilized hamster eggs is related to meiotic maturation of the maternal chromatin. J. exp. Zool. 247, 155-165.

Zirkin, B.R., Soucek, D.A., Chang, T.S.K. \& Perreault, S.D. (1985) In vitro and in vivo studies of mammalian sperm nuclear decondensation. Gamete Res. 11, 349-365.

Received 3 January 1990 